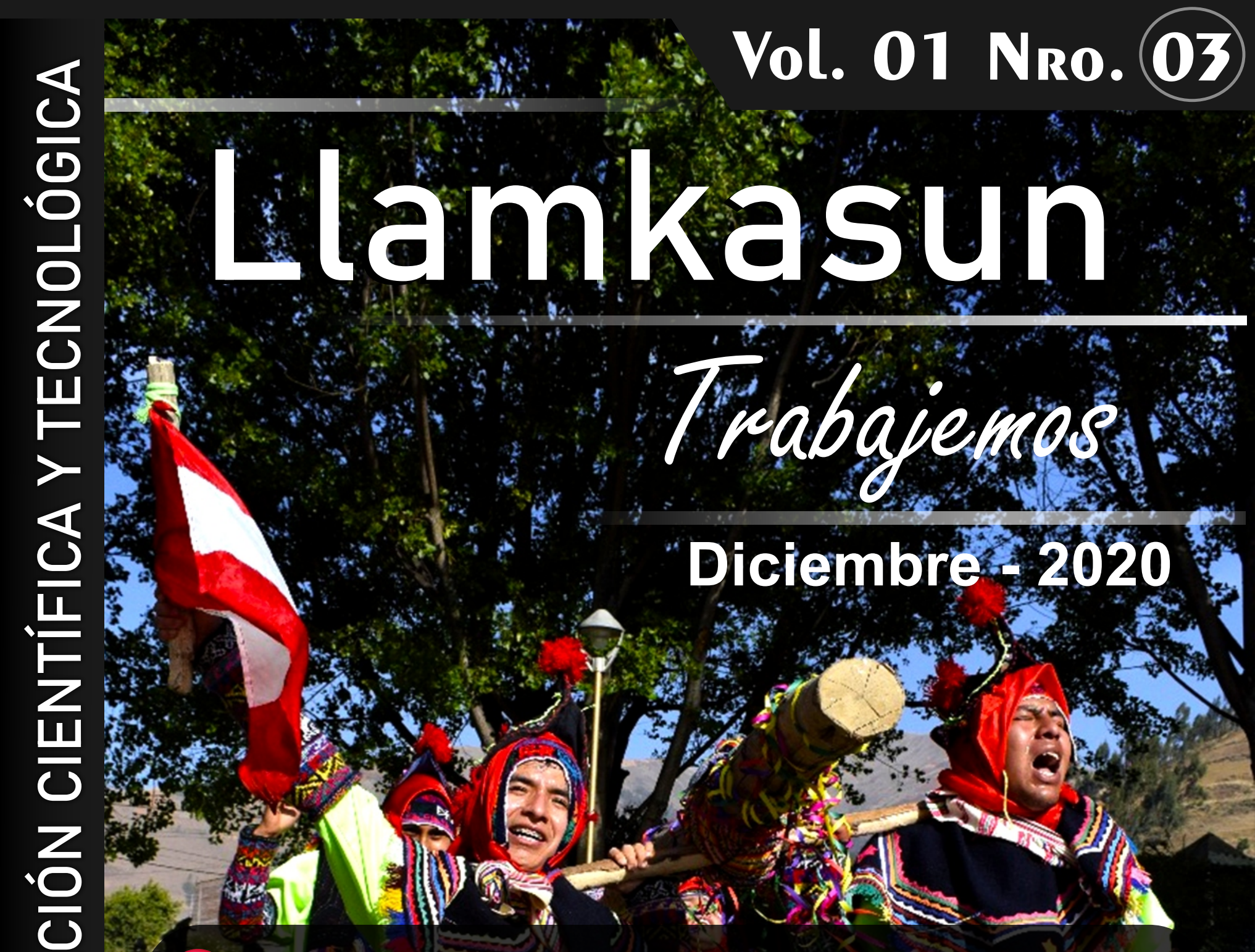




\title{
Elasticidad del gasto público en Perú periodo 1980-2018
}

\section{Elasticity of public spending in Peru, period 1980-2018}

\section{Elasticidade dos gastos públicos no Peru, período 1980-2018}

\author{
Alberto Valenzuela Muñoz \\ Universidad Nacional Autónoma de Tayacaja Daniel Hernández Morillo. Perú. \\ Carlos Alberto Choquehuanca Saldarriaga \\ Universidad César Vallejo. Perú. \\ Jose Torres Huamani \\ Universidad Nacional Autónoma de Tayacaja Daniel Hernández Morillo. Perú. \\ Madely Kaqui Valenzuela \\ Universidad Nacional Santiago Antúnez de Mayolo. Huaraz, Perú. \\ Sandra Elizabeth Huaman Pastorelli \\ Universidad San Martin de Porres. Lima, Perú.
}

\section{RESUMEN}

El objetivo del artículo ha sido determinar si la Ley de Wagner se cumple en el Perú durante el periodo 1980-2018. La Ley de Wagner propone que el crecimiento del Gasto Público se explica por el crecimiento del PBI. En la medida que existen distintas formas funcionales para estimar dicha relación, hemos tomados las tres primeras versiones de las seis que sintetiza Jaén (2014) para la economía española y las hemos estimado para el caso peruano. La primera versión que propone que el Gasto Público es una función del PBI; la segunda versión que propone que el Consumo Público es una función del PBI y la tercera que propone que el Gasto Público es una función del PBI per cápita. En ese sentido, hemos tomado datos reales sobre dichas variables publicados en la web del Banco Central de Reserva del Perú, se ha analizado su evolución y hemos estimado las tres formas funcionales propuestas. Las estimaciones prueban que, en las tres versiones analizadas, se cumple la Ley de Wagner en el Perú para el periodo 1980-2018.

Palabras clave: elasticidad, gasto, público, consumo, PBI 


\begin{abstract}
The objective of the article has been to determine if Wagner's Law is fulfilled in Peru during the period 1980-2018. Wagner's Law proposes that the growth of Public Expenditure is explained by the growth of the GDP. To the extent that there are different functional ways to estimate this relationship, we have taken the first three versions of the six that Jaén (2014) synthesizes for the Spanish economy and we have estimated them for the Peruvian case. The first version that proposes that Public Expenditure is a function of GDP; the second version that proposes that Public Consumption is a function of GDP and the third that proposes that Public Expenditure is a function of GDP per capita. In this sense, we have taken real data on these variables published on the website of the Central Reserve Bank of Peru, their evolution has been analyzed and we have estimated the three proposed functional forms. The estimates prove that, in the three versions analyzed, Wagner's Law is fulfilled in Peru for the period 1980-2018.
\end{abstract}

Keywords: elasticity, spending, public, consumption, GDP.

\title{
RESUMO
}

O objetivo do artigo foi determinar se a Lei de Wagner foi cumprida no Peru durante o período 1980-2018. A Lei de Wagner propõe que o crescimento da Despesa Pública seja explicado pelo crescimento do PIB. Na medida em que existem diferentes formas funcionais de estimar essa relação, tomamos as três primeiras versões das seis que Jaén (2014) sintetiza para a economia espanhola e as estimamos para o caso peruano. A primeira versão que propõe que a despesa pública é uma função do PIB; a segunda versão que propõe que o Consumo Público é função do PIB e a terceira que propõe que a Despesa Pública é função do PIB per capita. Nesse sentido, tomamos dados reais sobre essas variáveis publicados no site do Banco Central de Reserva do Peru, analisamos sua evolução e estimamos as três formas funcionais propostas. As estimativas comprovam que, nas três versões analisadas, a Lei de Wagner foi cumprida no Peru para o período 1980-2018.

Palavras-chave: elasticidade, gastos, público, consumo, PIB. 


\section{INTRODUCCIÓN}

En julio del año 1980 el Gobierno Militar, que se había iniciado en el año 1968, derrocando a Fernando Belaúnde Terry, devolvió, previa elaboración de una nueva Constitución Política del Perú, al electo, por segunda vez, Fernando Belaúnde Terry.

No vamos a considerar los doce años de la dictadura militar porque en dicho periodo las decisiones de los gobernantes (el ejecutivo que se dice), eran muy propias de la élite que ostentaba el poder, sino a partir de los gobiernos democráticos (que se dicen) que retomaron el control a partir del año 1980. Además, considerando las condiciones de Wagner necesarias para el cumplimiento de la ley (Incremento de la renta nacional y del bienestar general e inclusive de la renta per cápita, importancia del progreso tecnológico y Estado constitucional y democratización: participación de la población en las decisiones político financieras) (Jaén 2014, p. 18), lo más adecuado es tomar dicho periodo.

El segundo gobierno de Belaúnde inició un proceso de liberalización de la política comercial, que terminó en un rotundo fracaso para la economía; fue seguido por el gobierno de Alan García, cuyo manejo, al menos en sus primeros años, fue considerado heterodoxo, porque pasó del enfoque de la inflación por costos al de la inflación por demanda y que se hizo famoso por proponer restringir el pago de la deuda pública a no más del $10 \%$ del valor de las exportaciones, rompiendo con el FMI y pretendiendo estatizar la banca, de lo cual rápidamente se arrepintió. Luego vendría Fujimori, quien ganó contraponiéndose a la política de choque propuesta por su oponente Vargas LLosa, pero que terminó implementando dicha política a partir del 8 de agosto de 1990 con un dramático mensaje del entonces Ministro de Economía Juan Carlos Hurtado Miller, centrado en corregir los precios relativos y eliminar los subsidios, que hizo que, ese solo día, los precios se elevaran $400 \%$ en promedio. Luego, siguiendo las recetas del FMI, sintetizadas en el llamado Consenso de Washington, trataron de vender las empresas públicas y desprenderse de las funciones del gobierno sobre educación, salud y trabajo, con tal de reducir el aparato del Estado, eso sí Boloña (1993) propuso obtener dinero "fresco" para la economía, lo que lo condujo a reinsertarse en el sistema financiero internacional, lo cual los llevó a cambiar la deuda por los bonos Brady. Desde entonces, en el campo económico, 
las cosas han ocurrido por inercia, así que no vale la pena comentar los sucesivos gobiernos de Toledo, García, Humala, Kuczynski y Vizcarra. Si hay algo novedoso en lo que va del siglo XXI son los Tratados de Libre Comercio, que comenzaron con el Tratado de Libre Comercio de América del Norte (NAFTA por sus siglas en inglés) y se han extendido al mundo, enmascarando una política comercial de apertura.

El punto es que se ha generado un "debate" entre los que sostienen la vigencia de la llamada Ley de Wagner y la propuesta keynesiana sobre el papel del Estado en la economía, más concretamente de la relación de causalidad entre gasto y público y crecimiento económico. Así mientras la Ley de Wagner sostiene que el crecimiento del estado es resultado del crecimiento de la economía, Keynes cree que es el gasto público el que crea las condiciones para el crecimiento económico, llevando a cabo una política anticíclica.

Y como hay datos para todos los gustos y la matemática, la estadística y la econometría operadas ahora electrónicamente, pueden probar lo que les pidas, encontramos infinidad de trabajos en los cuales unos prueban una cosa y otros otra, con solo invertir el sentido de los datos: la variable explicada pasa a ser explicativa y así tenemos dos regresiones para los mismos datos.

La pregunta es ¿Será posible que en algunos años el crecimiento de la producción jale al aumento del gasto público y, en otros años, el gasto público sea el que impulse el crecimiento de la producción? Porque con una o con otra regresión, la correlación entre ambos va a ser la misma. Así que tal vez estamos en una supuesta discusión teórica sobre cosas que son obvias $\mathrm{y}$, en realidad, lo más interesante y útil es medir el grado de asociación. Quizás tengamos que referirnos a determinados momentos del ciclo económico, a determinados años o determinamos momentos en que una variable empuja a la otra, eso en el corto o mediano plazo. Otra historia es el largo plazo en el cual hay dos opciones: o caminan juntas o no caminan juntas, más allá que en el corto o mediano plazo tengan un grado de asociación o no.

Al respecto se han desarrollado diversas investigaciones a lo largo del tiempo, algunas de las cuales glosaremos.

Gandhi (1971) no hace un desarrollo propiamente empírico de la ley de Wagner, sino que se limita a entenderla como "la relación entre los gastos públicos y el nivel de desarrollo económico" (p. 78), a resumir las distintas formas como se ha entendido, para diversas investigación el sentido 
empírico de dicha relación y a concluir que "la relación funcional que más ha sido comprobada, con la ayuda de los datos de análisis por secciones transversales, es la que relaciona la participación del gasto público E/GNP con el nivel de desarrollo económico GNP/P” (p. 80), donde E es el nivel del gasto público, GNP es el Producto Nacional Bruto y P es la población. Y frente a la diversidad de resultados, le parece consistente "el hallazgo empírico de una significativa relación positiva (entre la participación de los gastos públicos y el producto nacional per cápita) en los estudios que se refieren a muestras de países tanto desarrollados como subdesarrollados" tanto como "la falta de tal relación en las experiencias de países subdesarrollados solamente" lo que en su opinión "sugiere que quizá la composición de muestras tiene algo que ver con los hallazgos de los análisis por secciones transversales en la validez de la ley de Wagner" (p. 88).

Uribe (2000) muestra, que más allá de los debates existentes, el hecho es que las razones que explican la participación del estado en la formación de la producción interna son muchas y que, en todo caso, el hecho es que, en todos los países del mundo, dicha participación ha estado creciendo y, en su opinión, “El Problema, más bien, está en cómo volverlo eficiente y eficaz, cómo hacer que garantice el bienestar colectivo con el mínimo de costo, o diciéndolo de otra manera, cómo lograr una buena gobernabilidad, una gerencia pública de calidad total" (p. 85).

Pérez (2009) hace un análisis más bien cualitativo y, en ese sentido, sostiene que "La tesis de la realización progresiva de los derechos económicos y sociales es una respuesta al problema de cómo relacionar la ampliación del ámbito de tales derechos con los recursos disponibles para atenderlos". De manera que "No se trata de un simple ejercicio de cuantificación sino de un proceso deliberativo que involucra, además, el cambio de valores y preferencias sociales que puede ilustrarse con la Ley de Wagner". Todo lo cual implica "la ampliación del conjunto de bienes o servicios meritorios; es decir, el proceso político lleva a definir los niveles de consumo mínimo hasta llegar a un ámbito básico, al que da el carácter de necesidad" (p. 268).

León (2017) prueba estadísticamente que, en el caso de Colombia en el periodo 19702010, “el gasto público ha crecido significativamente frente al ingreso per cápita” (p. 871) y considerando que "La ley de Wagner señala que en las etapas de preindustrialización y post-industrialización el crecimiento del gasto público debe ser menos que proporcional al PIB-percapita" 
(p. 872), recomienda que "el Gobierno aumente esfuerzos para invertir en gasto social que implique mayor bienestar de la población” (p. 873).

Campo y Mendoza (2018), sostiene que, en el caso de Colombia, durante el periodo 1984-2012, "Los resultados muestran un efecto positivo y significativo del gasto público primario sobre el PIB, lo cual va en la vía del enfoque keynesiano y confirma la hipótesis de que aumentos en el gasto público contribuyen al crecimiento económico" (p. 78).

Bulacio (2000) estudia la evolución del gasto público en Argentina en el período 1960-1999 y llega, entre otras, a la conclusión que "la elasticidad parcial del gasto con respecto al ingreso estimada para el período de 0,87 es muy superior a la que requeriría la estabilidad en la participación relativa del gasto público sobre el ingreso" (p. 15).

Perrotini (2002), en su artículo rinde un "merecido tributo a José Ayala Espino, quien fuera primus inter pares e insigne neoinstitucionalista de nuestro claustro universitario. $\mathrm{Su}$ vida y su obra evocan el primer aforismo de Hipócrates: Ars longa, vita brevis" (p. 29) y como resultado del neoinstitucionalismo, concluye que "El nudo gordiano parece estribar, al menos en parte, $[\ldots]$ en la naturaleza obsoleta $y$ disfuncional de los actuales "fundamentos institucionales del mercado". Lo cual, a su vez impediría que "el Estado en el tercer mundo opere la histórica tarea dual de creación y regulación del mercado [...] y que la competencia sea un factor detonante del progreso capitalista”, en razón de lo cual “Tributario del mejor keynesianismo [...], Pepe propone la actualización del marco institucional como antídoto para superar las antinomias del capitalismo global, i.e., el creciente desempleo estructural, el crecimiento insuficiente, la inestabilidad financiera y la pobreza (p. 33).

Díaz (2009) destaca la importancia del gasto público, considerándolo "el principal instrumento de política económica que el gobierno posee para atender las demandas de la sociedad. [lo cual] es el resultado lógico y natural de la existencia de necesidades públicas que generalmente son atendidas por decisiones políticas" (p. 1). Por lo cual, "El presupuesto debe responder a las necesidades del Estado e ir dirigido primordialmente a reducir la gran desigualdad social que impera en este país [México]". Por lo que propone elaborar un presupuesto por resultados que "implicaría formularlo considerando los objetivos nacionales y las necesidades de política económica reales" y, adicionalmente, “debe de instrumentarse un sistema de costes, y en 
cada etapa del proceso efectuar una evaluación integral del gasto público, no sólo al final y un año después y en algunos programas sociales y de desarrollo rural" ( $p$. $6)$.

Rodríguez, Venegas-Martínez y Lima (2013), en su investigación, examinan "la ley de Wagner y la hipótesis keynesiana para el caso de México durante el periodo 1950-2009" (p. 69). Y encuentra que "los distintos indicadores que relacionan el gasto público con el crecimiento económico efectivamente cointegran. Es decir, guardan entre ellos una relación de largo plazo mediante trayectorias comunes" (p. 92). Y, en cuanto a las pruebas de causalidad sostienen que "la evidencia empírica es robusta para validar el cumplimiento de la ley de Wagner, en detrimento de la hipótesis keynesiana" (p. 93).

Díaz y Revuelta (2009), en su artículo, analizan "el cumplimiento de la ley de Wagner y la conjetura keynesiana en España y América Latina desde 1960 hasta 2000” (p. 1), llegando, entre otras, a la conclusión que "En España, en lugar de la ley de Wagner, debemos aceptar la conjetura keynesiana, dado que es el incremento del gasto público el que causa el crecimiento económico" (p. 23). En las economías latinoamericanas, “fijándonos en las elasticidades tenemos motivos para afirmar que la ley se cumple en catorce de los diecinueve estados, siendo las excepciones Perú, Chile, República Dominicana, El Salvador y Nicaragua" (p. 24).

Sánchez (2009) en un artículo de crítica al manejo del gobierno, sostiene que la Ley de Wagner, se ha cumplido en el caso español, porque "la inversión pública en infraestructuras se situó en 2007 en el 3,8\% del Producto Interior Bruto, lo que representa el $147 \%$ de la media de la eurozona. Y 20 puntos de PIB más que la privada" (p. 1), "Pero que menudo se olvida la atrofia que supone tener un sector público altamente ineficiente" (p. 2).

Jaén (2014) realiza una revisión exhaustiva de la manera como se ha venido abordando el estudio empírico de la ley de Wagner llegando a la conclusión que "Los resultados obtenidos en los contrastes son muy diversos, el análisis de diversos autores ha puesto de manifiesto que no es sorprendente que los contrastes econométricos requeridos para examinar la ley de Wagner no sean concluyentes" (p. 16). Y señala que la inconsistencia de los resultados obtenidos por diferentes autores podría deberse a "1) la calidad y cantidad de los datos; 2) los procedimientos de contraste y el período examinado; 3) el nivel escogido de agregación temporal; 4) la especificación 
econométrica y 5) la influencia de variables omitidas" (pp. 16-17). Llegando a la conclusión que de acuerdo con las investigaciones más recientes el resultado más frecuente "es la bicausalidad o causalidad bidireccional. Es decir, ambas variables son endógenas, ninguna es débilmente exógena y, por tanto, ambas se influyen mutuamente de forma que resulta imposible determinar la línea de política adecuada". Para el caso español se ha tomado el periodo 1960-1995, utilizando las seis versiones que el autor resume como las más usuales, En este trabajo se han contrastado seis versiones empíricas de la ley de Wagner, resultando que "Si consideramos el crecimiento económico como un factor exógeno, el gasto público crecerá como consecuencia del crecimiento económico cualquiera que sea la forma en que éste se mida (aunque siempre en relación con el PIB)". Por el contrario "un aumento del gasto público no va a determinar (a preceder sería el término adecuado) el crecimiento económico" (p. 29).

Molina y Gantier (2015) realizan "una revisión del enunciado de la ley de Wagner y la teoría Keynesiana sobre gasto público en Bolivia durante el período 1990 hasta 2014” (p. 1), llegando a la conclusión que "el Gasto de Gobierno causa en el sentido de Granger al Producto Interno Bruto y no así viceversa en el corto plazo. Mientras que la causalidad de ambas variables en el largo plazo es nula (p. 14).

Feijóo y Peralta (2017) en su tesis analizan "la relación entre el Gasto público y el crecimiento económico para los países de la Comunidad Andina mediante un modelo de vectores autorregresivos (VAR), tanto en forma individual para cada país, así como también en datos de panel (PVAR)" (p. 2) para el periodo 1985-2015. La conclusión es que "existe evidencia empírica de bidireccionalidad para los modelos de $\mathrm{P} \& \mathrm{~W}$, Goffman y Gupta mientras que para el modelo de Mann y Musgrave se cumple la teoría de Keynes". Para Colombia "hemos encontrado evidencia a favor de Keynes para todos los modelos especificados", en el caso de "Ecuador en todas las interpretaciones se cumple la ley de Wagner a excepción de P\&W y en el caso de Perú no se ha encontrado evidencia a favor de ninguna de las dos teorías" (p. 152).

Aparco y Flores (2019) se propusieron "verificar el cumplimiento de la "Ley de Wagner" frente a la hipótesis keynesiana del gasto público en el Perú” (p. 53). Para "La contrastación empírica de la relación entre el gasto público y el crecimiento económico se realizará en el periodo 
comprendido entre 1950 y 2016, utilizando series anuales de estas variables transformadas en logaritmos naturales (p. 58). Los resultados de la investigación, validan en el largo plazo la Ley de Wagner en todas las versiones especificadas, "ya que se evidencia una relación de causalidad unidireccional del crecimiento económico al gasto público, además que todas las versiones cumplen con el criterio de elasticidad". Y en el corto plazo, en las versiones de Peacock y Wiseman (1961) y Mann (1980), “se cumple la hipótesis keynesiana de que el gasto público genera el crecimiento económico, a un nivel de significancia del $10 \%$; en las tres especificaciones restantes no se evidencia relación de causalidad en el corto plazo" ( $p$. 69).

Izquierdo, Puig, Riera-Crichton y Vuletin (2018) sostienen en la publicación del Banco Interamericano de Desarrollo que la participación de los gobiernos en la economía, desde inicio de la década de los 90 ha "aumentado de manera sostenida en todo el mundo". Y que la participación del gobierno en los países desarrollados "es casi dos veces superior a la de los países en desarrollo, incluida América Latina y el Caribe $(40 \%$ vs. $20 \%$ del PIB, respectivamente)". Lo que términos de la ley de Wagner, significa que "a medida que aumenta el PIB per cápita, el gasto público tiende a elevarse, tanto en el margen extensivo (aparecen actividades y servicios nuevos) como en el margen intensivo (se amplían las actividades y servicios existentes)" (p. 1).

\section{METODOLOGÍA}

Para llevar a cabo la investigación se reunió datos reales publicados por el Banco Central de Reserva del Perú en su página web, sobre las variables de investigación, considerando el periodo 1980-2018. Dichos datos están expresados en soles a precios constantes del año 2007. Para las estimaciones de la Ley de Wagner en el caso peruano, hemos tomado como referencia el trabajo de Jaén (2014), quien hace una síntesis de las principales versiones de dicha Ley y las aplica para el periodo 1960-1995 en España. Así adaptando dichas versiones a la simbología de las variables que usamos en el Perú, tendríamos las siguientes versiones de la ley de Wagner para el periodo 1980-2018:

La primera versión es atribuida a Peacock y Wisseman (1961), Musgrave (1969) y Goffman y Mahar (1971) y considera que el Gasto Público crece más rápidamente que el Producto Bruto Interno, de manera que la Elasticidad del Gasto Público, respecto del 
PBI, es mayor que 1. Así la función sería $G$ $=\mathrm{f}(\mathrm{PBI})$, donde $\mathrm{G}$ es el Gasto Público y PBI es el Producto Bruto Interno.

La segunda versión es atribuida a Pryor (1968) y considera que, si la economía está creciendo, el Consumo Público, expresado como proporción del PBI, también crece, de manera que la Elasticidad del Consumo Público, respecto del PBI, es mayor que 1. Así la función sería $\mathrm{C}=\mathrm{f}(\mathrm{PBI})$, donde $\mathrm{C}$ es el Consumo Público.

La tercera versión es atribuida a Goffman (1968) y considera que, argumenta que el crecimiento económico genera un aumento del sector público, lo que implica que el gasto público, crecerá a una tasa mayor que el PBI per cápita, de manera que la Elasticidad del Gasto Público, respecto del PBI per cápita, es mayor que 1. Así la función sería $G=f(P B I / P)$, donde $P$ es la población.

La cuarta versión es atribuida a Musgrave (1969) y considera que, en un país que pasa de una renta per cápita baja a una renta per cápita alta, se genera una proporción creciente del Gasto Público como proporción del PIB, de manera que la Elasticidad del Gasto Público cono proporción del PBI, respecto del PBI per cápita, es mayor que 1. Así la función sería $\mathrm{G} / \mathrm{PBI}=\mathrm{f}(\mathrm{PBI} / \mathrm{P})$.
La quinta versión es atribuida a Gupta (1967) y Michas (1975) y considera que, existe una relación entre el Gasto Público per cápita con el PBI per cápita, de manera que la Elasticidad del Gasto Público per cápita, respecto del PBI per cápita, es mayor que 1. Así la función sería $\mathrm{G} / \mathrm{P}=\mathrm{f}(\mathrm{PBI} / \mathrm{P})$. La sexta versión es atribuida a Peacock y Wisseman modificada por Mann (1980) y considera que, el Gasto Público como proporción del PBI es una función del PBI, de manera que la Elasticidad del Gasto Público como proporción del PBI, respecto del PBI, es mayor que 1. Así la función sería $\mathrm{GP} / \mathrm{PBI}=\mathrm{f}(\mathrm{PBI})$

En este caso, la idea es correr las regresiones para las tres primeras expresiones de la ley de Wagner sintetizadas por Jaén, para lo cual se regresará una variable en función de la otra, utilizando el método de cuadrados mínimos ordinarios al estilo del Teorema de GaussMarkov.

\section{RESULTADOS}

\section{Primera versión}

Hemos tomado datos reales del Gasto Público (G) y del Producto Bruto Interno (PBI) para el periodo 1980-2018, expresados en soles del año 2007, 
publicados por el Banco central de Reserva La figura 1 muestra la evolución de ambas del Perú (BCRP). variables.

La función a estimar es $G=f(P B I)$.

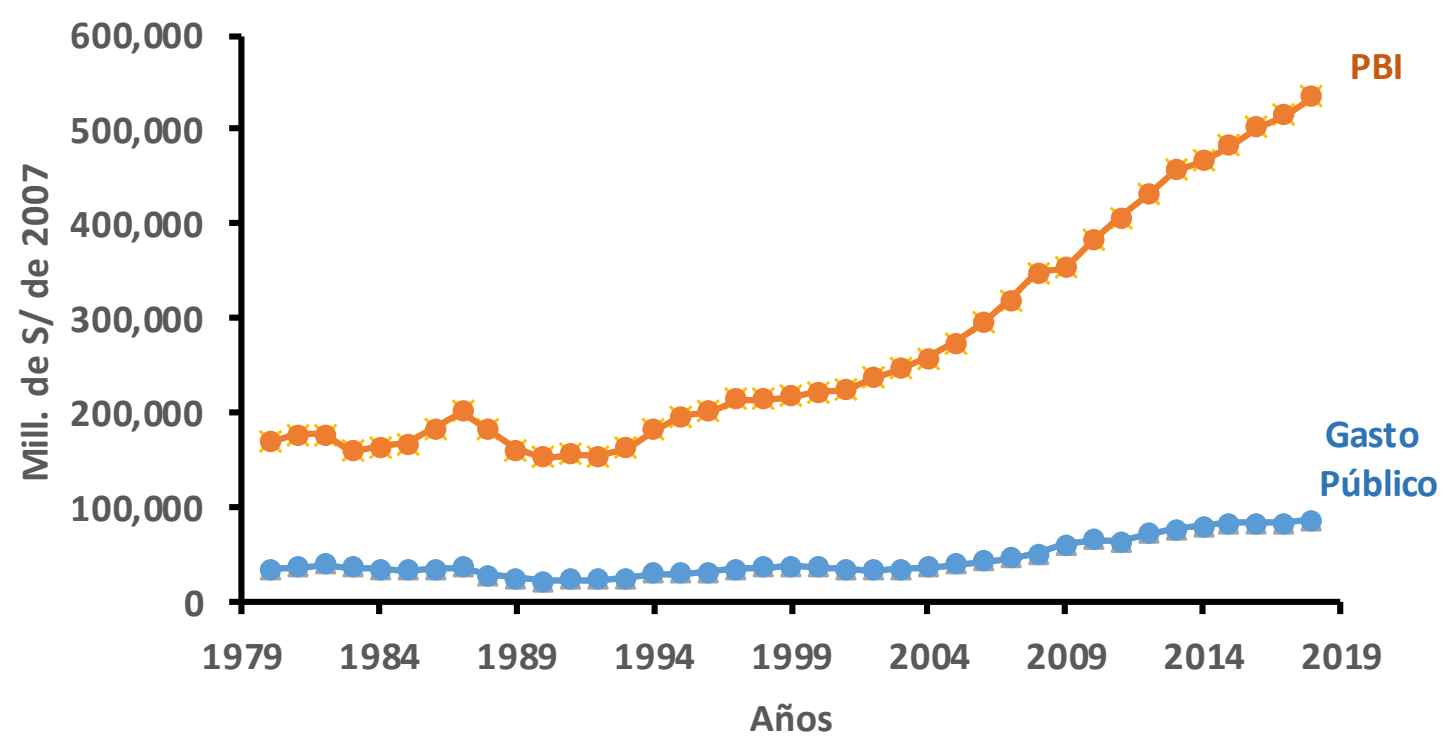

Figura 1. Evolución del Gasto Público del Perú 1980-2018, expresado en millones de soles del año 2007

Si estimamos la línea de tendencia del PBI, obtendremos los siguientes resultados:

$$
\begin{gathered}
\text { PBI }=-2 \mathrm{E}+07+9655.3 \mathrm{t} \\
\mathrm{r}^{2}=0.8265
\end{gathered}
$$

Que muestran una relación positiva del PBI con el tiempo (t), con un elevado nivel de ajuste de la regresión a los datos, expresado en el r2, lo que nos indica que hay una tendencia creciente del PBI en el periodo 1980-2018.
Igualmente, si estimamos la línea de tendencia del Gasto Público, obtendremos los siguientes resultados:

$$
G=-3 E+06+1400.4 t
$$

$$
r^{2}=0.6522
$$

Que muestran una relación positiva de $\mathrm{G}$ con $\mathrm{t}$, con un buen nivel de ajuste de la regresión a los datos, expresado en el r2, lo que nos indica que hay una tendencia creciente del G en el periodo 1980-2018.

De manera que podemos considerar como adecuada la forma lineal de la relación entre 
G y PBI, que quedaría expresada a través La tabla 1 muestra los resultados de la del siguiente modelo: regresión

$$
\mathrm{G}=\beta_{0}+\beta_{1} \mathrm{PBI}+\mu
$$

\section{Tabla 1}

Análisis de varianza de la regresión del Gasto Público y el PBI 1980-2018, expresados en soles constantes de 2007

\begin{tabular}{lccccc}
\hline Fuente & GL & SC Ajust. & MC Ajust. & Valor F & Valor p \\
\hline Regresión & 1 & $1.41 \mathrm{E}+22$ & $1.41 \mathrm{E}+22$ & 673.59 & 0.0000 \\
PBI & 1 & $1.41 \mathrm{E}+22$ & $1.41 \mathrm{E}+22$ & 673.59 & 0.000 \\
Error & 37 & $7.73 \mathrm{E}+20$ & $2.09 \mathrm{E}+19$ & & \\
Total & 38 & $1.49 \mathrm{E}+22$ & & & \\
\hline
\end{tabular}

Resumen del modelo

\begin{tabular}{ccc}
\hline$r^{2}$ & $r^{2}$ (ajustado) & $r^{2}$ pred \\
\hline $94.79 \%$ & $94.65 \%$ & $94.31 \%$ \\
\hline
\end{tabular}

Coeficientes

\begin{tabular}{lccccc}
\hline Término & Coef & EE del coef. & Valor T & Valor $\mathrm{p}$ & FIV \\
\hline Constante & 710082779 & 1809079808 & 0.39 & 0.697 & \\
PBI & 0.15896 & 0.00612 & 25.95 & 0.000 & 1 \\
\hline
\end{tabular}

\begin{tabular}{l} 
Ecuación de regresión \\
\hline $\mathrm{G}=710082779+0.15896 \mathrm{PBI}$ \\
\hline
\end{tabular}

Dichos resultados muestran que, con un nivel de seguridad del $95 \%$, hay una relación significativa entre $\mathrm{G}$ y $\mathrm{PBI}$, tanto en forma global como en forma individual, con un elevadísimo nivel de ajuste, expresado en un $\mathrm{r} 2$ con un nivel cercano al $95 \%$. Sin embargo, el valor del D-W es muy pequeño lo que es un indicativo de autocorrelación.
Estadístico de Durbin-Watson

Estadís tico de Durbin-Watson $=0.25265$
Siguiendo a Gujarati (2006), haremos las correcciones utilizando el test de D-W, suponiendo un modelo AR (1), ya que si se corrige la autocorrelación tendremos variables cointegradas que garantizan que, en el largo plazo, se puede establecer de manera estadísticamente significativa y no espuria la relación entre las variables. 
En primer lugar, la tabla 2 muestra los nos permitan corregir los datos originales de resultados de la regresión entre et y et-1, que G y PBI.

Tabla 2

Análisis de varianza de la regresión: $e_{t}$ vs. $e_{t-1}$

\begin{tabular}{lccccc}
\hline \multicolumn{1}{c}{ Fuente } & GL & SC Ajust. & MC Ajust. & Valor F & Valor p \\
\hline Regresión & 1 & $5.54 \mathrm{E}+20$ & $5.54 \mathrm{E}+20$ & 113.39 & 0.000 \\
$\mathrm{e}_{\mathrm{t}-1}$ & 1 & $5.54 \mathrm{E}+20$ & $5.54 \mathrm{E}+20$ & 113.39 & 0.000 \\
Error & 36 & $1.76 \mathrm{E}+20$ & $4.89 \mathrm{E}+18$ & & \\
Total & 37 & $7.30 \mathrm{E}+20$ & & & \\
\hline
\end{tabular}

Resumen del modelo

\begin{tabular}{ccc}
\hline$r^{2}$ & $r^{2}$ ajustado & $r^{2}$ pred \\
\hline $75.90 \%$ & $75.23 \%$ & $72.69 \%$ \\
\hline
\end{tabular}

Coeficientes

\begin{tabular}{lccccc}
\hline Término & Coef & EE del coef. & Valor $\mathrm{T}$ & Valor $\mathrm{p}$ & FIV \\
\hline Constante & -181738251 & 358590003 & -0.51 & 0.615 & \\
$\mathrm{e}_{\mathrm{t}-1}$ & 0.8465 & 0.0795 & 10.65 & 0.000 & 1 \\
\hline
\end{tabular}

Ecuación de regresión

$\mathrm{e}_{\mathrm{t}}=-181738251+0.8465 \mathrm{e}_{\mathrm{t}-1}$

\section{Estadístico de Durbin-Watson}

Estadístico de Durbin-Watson = 1.6193
Dichos resultados muestran una relación significativa entre los residuos, con una seguridad del $95 \%$ y que el $\mathrm{D}-\mathrm{W}=1.6193$, significa que no hay autocorrelación entre ellos.
La tabla 3 muestra los resultados con los datos corregidos de G y PBI usando $\rho=$ 0.8465 . 
Tabla 3

Análisis de varianza de la regresión de G y PBI corregidos

\begin{tabular}{lccccc}
\hline \multicolumn{1}{c}{ Fuente } & GL & SC Ajust. & MC Ajust. & Valor F & Valor $\mathrm{p}$ \\
\hline Regresión & 1 & $6.95 \mathrm{E}+20$ & $6.95 \mathrm{E}+20$ & 142.87 & 0.000 \\
PBI & 1 & $6.95 \mathrm{E}+20$ & $6.95 \mathrm{E}+20$ & 142.87 & 0.000 \\
Error & 36 & $1.75 \mathrm{E}+20$ & $4.87 \mathrm{E}+18$ & & \\
Total & 37 & $8.71 \mathrm{E}+20$ & & & \\
\hline
\end{tabular}

Resumen del modelo

\begin{tabular}{ccc}
\hline $\mathrm{r}^{2}$ & $\mathrm{r}^{2}$ ajustado & $\mathrm{r}^{2}$ pred \\
\hline $79.87 \%$ & $79.31 \%$ & $77.73 \%$ \\
\hline
\end{tabular}

Coeficientes

\begin{tabular}{lccccc}
\hline Término & Coef & EE del coef. & Valor T & Valor $\mathrm{p}$ & FIV \\
\hline Constante & -326178134 & 774641245 & -0.42 & 0.676 & \\
PBI corregido & 0.164 & 0.0137 & 11.95 & 0.000 & 1 \\
\hline
\end{tabular}

Ecuación de regresión

$\mathrm{G}=-326178134+0.1640$ PBI $\frac{\text { Estadístico de Durbin-Watson }}{\text { Estadístico de }}$

Para ver si la elasticidad PBI del Gasto Público es mayor que 1 , estimaremos una función logarítmica en las mismas condiciones que hemos estimados la regresión con los datos corregidos.

La ecuación a estimar sería la siguiente:

$$
\operatorname{Ln} G=\beta_{0}+\beta_{1} \operatorname{Ln} \text { PBI }
$$

Donde Ln es el logaritmo natural.

La tabla 4 muestra los resultados de la estimación de la función logaritmo natural. sentido, 0.16 céntimos, en promedio. 
Tabla 4

Análisis de varianza de la regresión del Ln GP y el Ln PBI corregidos

\begin{tabular}{lccccc}
\hline Fuente & GL & SC Ajust. & MC Ajust. & Valor F & Valor p \\
\hline Regresión & 1 & 0.167 & 0.167032 & 59.5 & 0.000 \\
Ln PBI & 1 & 0.167 & 0.167032 & 59.5 & 0.000 \\
Error & 36 & 0.1011 & 0.002807 & & \\
Total & 37 & 0.2681 & & & \\
\hline
\end{tabular}

Resumen del modelo

\begin{tabular}{ccc}
\hline $\mathrm{r}^{2}$ & $\mathrm{r}^{2}$ ajustado & $\mathrm{r}^{2}$ pred \\
\hline $62.30 \%$ & $61.26 \%$ & $57.24 \%$ \\
\hline
\end{tabular}

Coeficientes

\begin{tabular}{lccccc}
\hline Término & Coef & EE del coef. & Valor T & Valor $\mathrm{p}$ & FIV \\
\hline Constante & -0.221 & 0.199 & -1.11 & 0.274 & \\
Ln PBI & 1.084 & 0.14 & 7.71 & 0.000 & 1 \\
\hline
\end{tabular}

Ecuación de regresión

Ln GP $=-0.221+1.084 \mathrm{Ln}$ PBI

Estadístico de Durbin-Watson

Estadístico de Durbin-Watson $=1.56633$
Los resultados muestran que la elasticidad PBI del $G$ es mayor que 1 , con lo cual se estaría cumpliendo la Ley de Wagner.

\section{Segunda versión}

Hemos tomado datos reales del Consumo

Público (C) y del Producto Bruto Interno
(PBI) para el periodo 1980-2018, expresados en soles del año 2007, publicados por el Banco central de Reserva del Perú $\left(\mathrm{BC}_{\mathrm{RP}}\right)$. La función a estimar es $\mathrm{C}$ $=\mathrm{f}(\mathrm{PBI})$.

La figura 2 muestra la evolución del consumo público. 


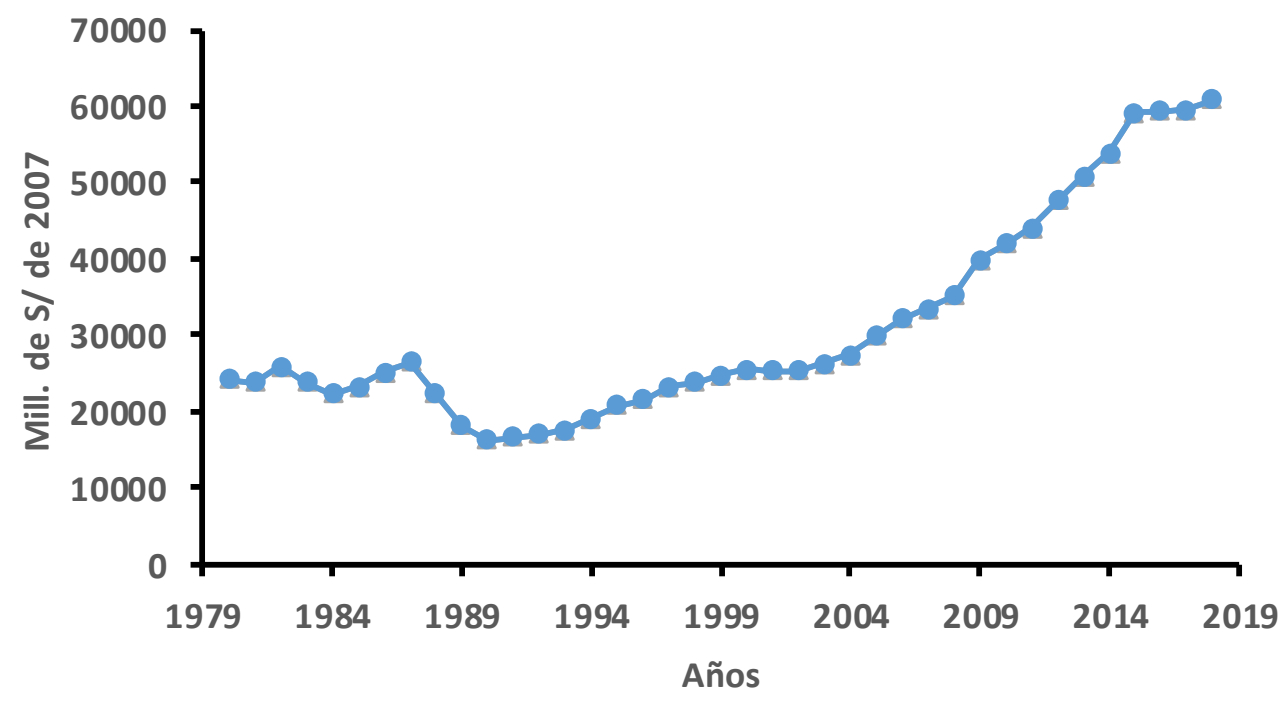

Figura 2. Evolución del Consumo Público del Perú 1980-2018, expresado en millones de soles del año 2007

Ya hemos visto que el PBI que muestra una tendencia creciente, si ahora estimamos la línea de tendencia del Consumo Público (C), obtendremos los siguientes resultados:

$\mathrm{C}=-2 \mathrm{E}+06+978.4 \mathrm{t}$

$\mathrm{r}^{2}=0.6836$

Que muestran una relación positiva de C con $\mathrm{t}$, con un buen nivel de ajuste de la regresión a los datos, expresado en el r2, lo que nos indica que hay una tendencia creciente del C en el periodo 1980-2018.

De manera que podemos considerar como adecuada la forma lineal de la relación entre C y PBI, que quedaría expresado a través del siguiente modelo:

$$
\mathrm{C}=\beta_{0}+\beta_{1} \mathrm{PBI}+\mu
$$

La tabla 5 muestra los resultados de la regresión. 
Tabla 5

Análisis de regresión del Consumo Público y el PBI, expresados en soles constantes de 2007

\begin{tabular}{lccccc}
\hline Fuente & GL & SC Ajust. & MC Ajust. & Valor F & Valor p \\
\hline Regresión & 1 & $6.67 \mathrm{E}+21$ & $6.67 \mathrm{E}+21$ & 1003.38 & 0.000 \\
PBI & 1 & $6.67 \mathrm{E}+21$ & $6.67 \mathrm{E}+21$ & 1003.38 & 0.000 \\
Error & 37 & $2.46 \mathrm{E}+20$ & $6.65 \mathrm{E}+18$ & & \\
Total & 38 & $6.92 \mathrm{E}+21$ & & & \\
\hline
\end{tabular}

Resumen del modelo

\begin{tabular}{ccc}
\hline$r^{2}$ & $r^{2}$ ajustado & $r^{2}$ pred \\
\hline 0.9644 & 0.9635 & 0.9604 \\
\hline
\end{tabular}

Coeficientes

\begin{tabular}{lccccc}
\hline \multicolumn{1}{c}{ Término } & Coef & EE del coef. & Valor T & Valor $\mathrm{p}$ & FIV \\
\hline Constante & 1546635846 & 1020279866 & 1.52 & 0.138 & \\
PBI & 0.10942 & 0.00345 & 31.68 & 0.000 & 1 \\
\hline
\end{tabular}

Ecuación de regresión

$\mathrm{C}=1546635846+0.10942$ PBI
Estadístico de Durbin-Watson

Estadístico de Durbin-Watson $=0.293634$
Dichos resultados muestran que, con un nivel de seguridad del $95 \%$, hay una relación significativa entre $\mathrm{C}$ y $\mathrm{PBI}$, tanto en forma global como en forma individual, con un elevadísimo nivel de ajuste, expresado en un $\mathrm{r} 2$ con un nivel superior al $96 \%$. Sin embargo, el valor del D-W es muy pequeño lo que es un indicativo de autocorrelación.

Como en el modelo anterior, se requiere, entonces, hacer las correcciones necesarias para que las estimaciones no sean espurias, así que suponiendo un modelo AR (1), ya que si se corrige la autocorrelación tendremos variables cointegradas que garantizan que, en el largo plazo, se puede establecer de manera estadísticamente significativa y no espuria la relación entre las variables.

En la tabla 6 se muestran los resultados de la regresión entre et y et-1, que nos permitan corregir los datos originales de $\mathrm{C}$ y $\mathrm{PBI}$. 
Tabla 6

Análisis de varianza de la regresión de e $t$ y.e $e_{t-1}$

\begin{tabular}{lccccc}
\hline Fuente & GL & SC Ajust. & MC Ajust. & Valor F & Valor p \\
\hline Regresión & 1 & $1.63 \mathrm{E}+20$ & $1.63 \mathrm{E}+20$ & 92.55 & 0.000 \\
et-1 & 1 & $1.63 \mathrm{E}+20$ & $1.63 \mathrm{E}+20$ & 92.55 & 0.000 \\
Error & 36 & $6.36 \mathrm{E}+19$ & $1.77 \mathrm{E}+18$ & & \\
Total & 37 & $2.27 \mathrm{E}+20$ & & & \\
\hline
\end{tabular}

Resumen del modelo

\begin{tabular}{ccc}
\hline $\mathrm{r}^{2}$ & $\mathrm{r}^{2}$ ajustado & $\mathrm{r}^{2}$ pred \\
\hline $71.99 \%$ & $71.22 \%$ & $68.23 \%$
\end{tabular}

Coeficientes

\begin{tabular}{lccccc}
\hline \multicolumn{1}{c}{ Término } & Coef & EE del coef. & Valor $\mathrm{T}$ & Valor $\mathrm{p}$ & FIV \\
\hline Constante & -97986322 & 215593188 & -0.45 & 0.652 & \\
$\mathrm{e}_{\mathrm{t}-1}$ & 0.816 & 0.0848 & 9.62 & 0.000 & 1 \\
\hline
\end{tabular}

Ecuación de regresión

$e_{t}=-97986322+0.8160 e_{t-1}$
Estadístico de Durbin-Watson

Estadístico de Durbin-Watson $=1.88128$
Dichos resultados muestran una relación significativa entre los residuos, con una seguridad del $95 \%$ y que el $\mathrm{D}-\mathrm{W}=$ 1.88128, significa que no hay autocorrelación entre ellos.
La tabla 7 muestra los resultados con los datos corregidos de C y PBI usando $\rho=$ 0.8160 . 
Tabla 7

Análisis de varianza de la regresión del Consumo Público y el PBI corregidos

\begin{tabular}{lccccc}
\hline Fuente & GL & SC Ajust. & MC Ajust. & Valor F & Valor p \\
\hline Regresión & 1 & $4.15 \mathrm{E}+20$ & $4.15 \mathrm{E}+20$ & 236.5 & 0.000 \\
PBI corregido & 1 & $4.15 \mathrm{E}+20$ & $4.15 \mathrm{E}+20$ & 236.5 & 0.000 \\
Error & 36 & $6.32 \mathrm{E}+19$ & $1.75 \mathrm{E}+18$ & & \\
Total & 37 & $4.78 \mathrm{E}+20$ & & & \\
\hline
\end{tabular}

Resumen del modelo

\begin{tabular}{ccc}
\hline $\mathrm{r}^{2}$ & $\mathrm{r}^{2}$ ajustado & $\mathrm{r}^{2}$ pred. \\
\hline $86.79 \%$ & $86.42 \%$ & $85.08 \%$ \\
\hline
\end{tabular}

Coeficientes

\begin{tabular}{lccccc}
\hline Término & Coef & EE del coef. & Valor T & Valor $\mathrm{p}$ & FIV \\
\hline Constante & -18360296 & 477694181 & -0.04 & 0.97 & \\
PBI corregido & 0.11295 & 0.00734 & 15.38 & 0.000 & 1 \\
\hline
\end{tabular}

Ecuación de regresión Ccorreg $=-18360296+0.11295$ PBIcorreg

Dichos resultados muestran que, con un nivel de seguridad del $95 \%$, hay una relación significativa entre $\mathrm{C}$ y $\mathrm{PBI}$, tanto en forma global como en forma individual, con un elevado nivel de ajuste, expresado en un r2 con un nivel cercano al $87 \%$ y, por otra parte, el D-W = 1.92637 indica ausencia de autocorrelación.

Así, que, en principio podemos concluir que, por cada sol que ha variado el PBI, el consumo público ha variado en el mismo sentido, 0.11 céntimos, en promedio.
Estadístico de Durbin-Watson

Estadístico de Durbin-W atson $=1.92637$
Donde Ln es el logaritmo natural.

La tabla 8 muestra los resultados de la estimación de la función logarítmica. 
Tabla 8

Análisis de varianza de la regresión del Ln C y Ln PBI corregidos

\begin{tabular}{lccccc}
\hline Fuente & GL & SC Ajust. & MC Ajust. & Valor F & Valor p \\
\hline Regresión & 1 & 0.2727 & 0.272696 & 153.84 & 0.000 \\
Ln PBI corregi & 1 & 0.2727 & 0.272696 & 153.84 & 0.000 \\
Error & 36 & 0.06381 & 0.001773 & & \\
Total & 37 & 0.33651 & & & \\
\hline
\end{tabular}

Resumen del modelo

\begin{tabular}{ccc}
\hline$r^{2}$ & $r^{2}$ ajustado & $r^{2}$ pred. \\
\hline $81.04 \%$ & $80.51 \%$ & $78.18 \%$ \\
\hline
\end{tabular}

Coeficientes

\begin{tabular}{lccccc}
\hline \multicolumn{1}{c}{ Término } & Coef & EE del coef. & Valor T & Valor $\mathrm{p}$ & FIV \\
\hline Constante & -0.329 & 0.3 & -1.1 & 0.279 & \\
Ln PBI & 1.0059 & 0.0811 & 12.4 & 0.000 & 1 \\
\hline
\end{tabular}

Ecuación de regresión

Ln C $=-0.329+1.0059$ Ln PBI
Estadístico de Durbin-Watson

Estadístico de Durbin-Watson $=1.89536$
Los resultados muestran que la elasticidad PBI del $\mathrm{C}$ es mayor que 1 , con lo cual se estaría cumpliendo la Ley de Wagner.

Tercera versión

Lo nuevo en esta versión es el PBI per cápita, que hemos obtenido tomando los datos de la población publicados por el Banco central de Reserva del Perú (BCRP). La función a estimar es

$\mathrm{G}=\mathrm{f}(\mathrm{PBI} /$ Población $)=\mathrm{f}($ PBI per cápita $)$.

La figura 3 muestra la evolución del PBI per cápita. 


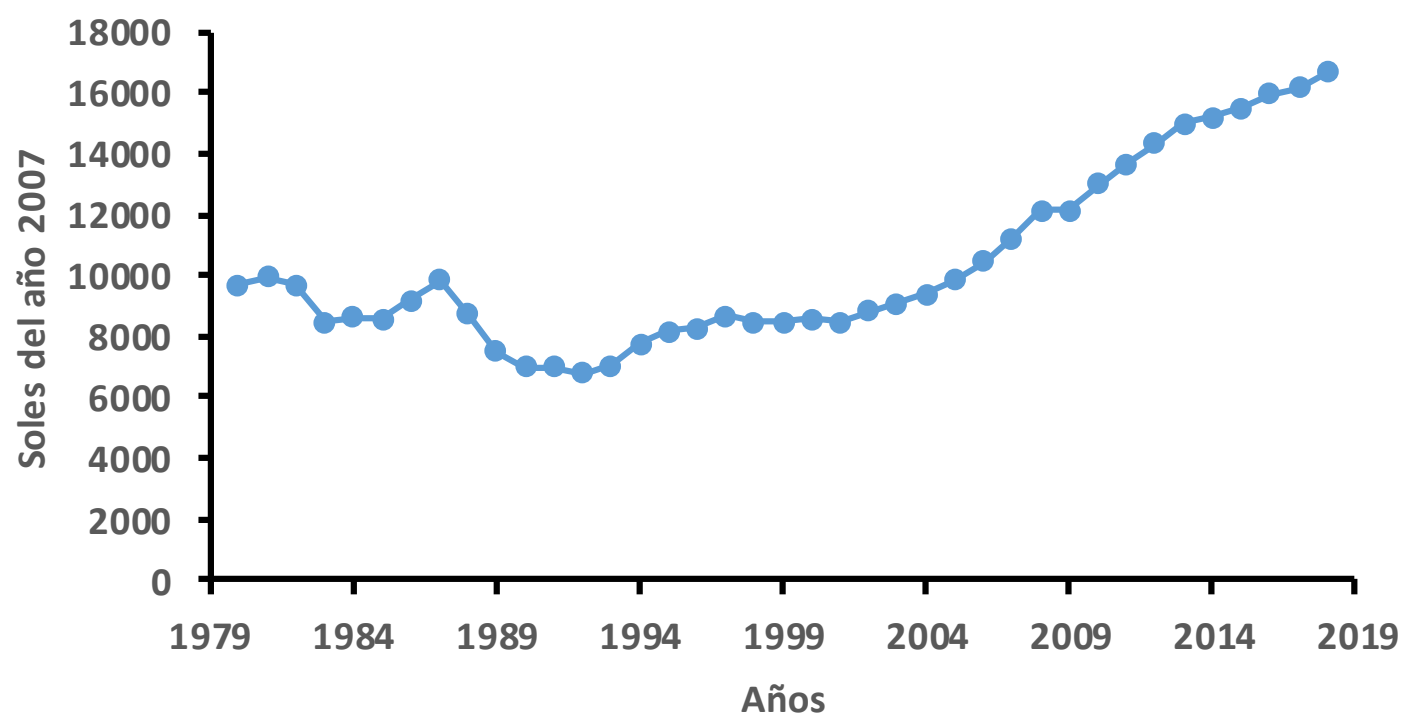

Figura 3. Evolución del PBI per cápita del Perú 1980-2018, expresado en soles del año 2007

Ya hemos visto que el PBI y el Gasto

Público muestran una tendencia creciente, si ahora estimamos la línea de tendencia del PBI per cápita (PBIPC), obtendremos los siguientes resultados:

$$
\begin{gathered}
\text { PBI }_{P C}=-391520+201.03 t \\
r^{2}=0.6159
\end{gathered}
$$

Que muestran una relación positiva del PBIPC con t, con un buen nivel de ajuste de la regresión a los datos, expresado en el r2, lo que nos indica que hay una tendencia creciente del PBIPC en el periodo 19802018.

De manera que podemos considerar como adecuada la forma lineal de la relación entre G y PBI $_{\mathrm{PC}}$, que quedaría expresada a través del siguiente modelo:

$$
\mathrm{G}=\beta_{0}+\beta_{1} \mathrm{PBI}_{\mathrm{PC}}+\mu
$$

La tabla 9 muestra los resultados de la regresión. 
Tabla 9

Análisis de regresión del Gasto Público y el PBI per cápita, expresados en soles constantes de 2007

\begin{tabular}{lccccc}
\hline Fuente & GL & SC Ajust. & MC Ajust. & Valor F & Valor p \\
\hline Regresión & 1 & $1.45 \mathrm{E}+22$ & $1.45 \mathrm{E}+22$ & 1469.69 & 0.000 \\
PBI percápita & 1 & $1.45 \mathrm{E}+22$ & $1.45 \mathrm{E}+22$ & 1469.69 & 0.000 \\
Error & 37 & $3.65 \mathrm{E}+20$ & $9.86 \mathrm{E}+18$ & & \\
Total & 38 & $1.49 \mathrm{E}+22$ & & & \\
\hline
\end{tabular}

Resumen del modelo

\begin{tabular}{ccc}
\hline $\mathrm{r}^{2}$ & $\mathrm{r}^{2}$ ajustado & $\mathrm{r}^{2}$ pred \\
\hline 0.9754 & 0.9748 & 0.9732 \\
\hline
\end{tabular}

Coeficientes

\begin{tabular}{lccccc}
\hline \multicolumn{1}{c}{ Término } & Coef & EE del coef. & Valor T & Valor p & FIV \\
\hline Constante & -25450600000 & 1871170380 & -13.6 & 0 & \\
PBI percápita & 6685625 & 174393 & 38.340 & 0.000 & 1 \\
\hline
\end{tabular}

\section{Ecuación de regresión}

$\mathrm{G}=-25450564266+6685625 \mathrm{~PB} \mathrm{I}_{\mathrm{PC}}$

Dichos resultados muestran que, con un nivel de seguridad del $95 \%$, hay una relación significativa entre $\mathrm{C}$ y PBIPC, tanto en forma global como en forma individual, con un elevadísimo nivel de ajuste, expresado en un r2 con un nivel superior al $97 \%$. Sin embargo, el valor del $\mathrm{D}-\mathrm{W}$ es muy pequeño lo que es un indicativo de autocorrelación.

Como en el modelo anterior, se requiere, entonces, hacer las correcciones para
Estadístico de Durbin-Watson

Estadístico de Durbin-Watson $=0.7403$

corregir el problema, así que suponiendo un modelo AR (1), ya que si se corrige la autocorrelación tendremos variables cointegradas que garantizan que, en el largo plazo, se puede establecer de manera estadísticamente significativa y no espuria la relación entre las variables.

En la tabla 10 se muestran los resultados de la regresión entre et y et-1, que nos permitan corregir los datos originales de G y PBIPC. 
Tabla 10

Análisis de varianza de la regresión de $e_{t} y \cdot e_{t-1}$

\begin{tabular}{lccccc}
\hline Fuente & GL & SC Ajust. & MC Ajust. & Valor F & Valor p \\
\hline Regresión & 1 & $1.27 \mathrm{E}+20$ & $1.2701 \mathrm{E}+20$ & 21.96 & 0.000 \\
et-1 & 1 & $1.27 \mathrm{E}+20$ & $1.2701 \mathrm{E}+20$ & 21.96 & 0.000 \\
Error & 36 & $2.08 \mathrm{E}+20$ & $5.7841 \mathrm{E}+18$ & & \\
Total & 37 & $3.35 \mathrm{E}+20$ & & & \\
\hline
\end{tabular}

Resumen del modelo

\begin{tabular}{ccc}
\hline$r^{2}$ & $r^{2}$ ajustado & $r^{2}$ pred \\
\hline 0.3789 & 0.3616 & 0.2821 \\
\hline
\end{tabular}

Coeficientes

\begin{tabular}{lccccc}
\hline \multicolumn{1}{c}{ Término } & Coef & EE del coef. & Valor T & Valor p & FIV \\
\hline Constante & 133700440 & 390148299 & 0.34 & 0.734 & \\
et-1 & 0.59 & 0.126 & 4.69 & 0.000 & 1 \\
\hline
\end{tabular}

\section{Ecuación de regresión}

$\mathrm{e}_{\mathrm{t}}=133700440+0.590$ et -1

Dichos resultados muestran una relación significativa entre los residuos, con una seguridad del $95 \%$ y que el $\mathrm{D}-\mathrm{W}=1.6074$, significa que no hay autocorrelación entre ellos.

La tabla 7 muestra los resultados con los datos corregidos de G y PBIPC usando $\rho=$ 0.590 .

Tabla 11

Análisis de varianza de la regresión del Gasto Público y el PBI per cápita corregidos

\begin{tabular}{lccccc}
\hline Fuente & GL & SC Ajust. & MC Ajust. & Valor F & Valor p \\
\hline Regresión & 1 & $2.90 \mathrm{E}+21$ & $2.90 \mathrm{E}+21$ & 512.48 & 0.000 \\
PBIPC correg: & 1 & $2.90 \mathrm{E}+21$ & $2.90 \mathrm{E}+21$ & 512.48 & 0.000 \\
Error & 36 & $2.03 \mathrm{E}+20$ & $5.65 \mathrm{E}+18$ & & \\
Total & 37 & $3.10 \mathrm{E}+21$ & & & \\
\hline
\end{tabular}

Resumen del modelo

\begin{tabular}{|c|c|c|c|c|c|}
\hline$r^{2}$ & $\mathrm{r}^{2}$ ajustado & $r^{2}$ pred. & & & \\
\hline $93.44 \%$ & $93.25 \%$ & $92.78 \%$ & & & \\
\hline \multicolumn{6}{|l|}{ Coeficientes } \\
\hline Término & Coef & EE del coef. & Valor T & Valor $\mathrm{p}$ & FIV \\
\hline Constante & -9166400123 & 1294133919 & -7.08 & 0.000 & \\
\hline PBIPC corregic & 6424949 & 283813 & 22.64 & 0.000 & 1 \\
\hline
\end{tabular}

Ecuación de regresión

Estadístico de Durbin-Watson

Gcorreg $=-9166400123+6424949$ PBI $_{\mathrm{PC}}$ correg Estadístico de Durbin-Watson $=1.56568$
Estadístico de Durbin-Watson

Estadís tico de Durbin-Watson = 1.6074

Dichos resultados muestran que, con un nivel de seguridad del $95 \%$, hay una relación significativa entre $G$ y PBIPC, tanto en forma global como en forma individual, con un elevado nivel de ajuste, expresado en un $\mathrm{r} 2$ con un nivel superior al $93 \% \mathrm{y}$, por otra parte, el $\mathrm{D}-\mathrm{W}=1.56568$ indica ausencia de autocorrelación.

Así, que, en principio, podemos concluir que, por cada sol que ha variado el PBIPC, el Gasto Público ha variado en el mismo sentido, S/ 6,424,949, en promedio.

Para ver si la elasticidad PBI del Gasto Público es mayor que 1, estimaremos una función logarítmica en las mismas condiciones que hemos estimados la regresión con los datos corregidos. 
La ecuación a estimar sería la siguiente:

$\operatorname{Ln} G=\beta_{0}+\beta_{1} \operatorname{Ln}$ PBIPC

\begin{tabular}{|c|c|c|c|c|c|}
\hline \multicolumn{6}{|c|}{ Análisis de varianza de la regresión del Ln C y Ln PBI corregidos } \\
\hline Fuente & GL & SC Ajust. & MC Ajust. & Valor $\mathrm{F}$ & Valor $\mathrm{p}$ \\
\hline Regresión & 1 & 0.2727 & 0.272696 & 153.84 & 0.000 \\
\hline Ln PBI corregi & 1 & 0.2727 & 0.272696 & 153.84 & 0.000 \\
\hline Error & 36 & 0.06381 & 0.001773 & & \\
\hline Total & 37 & 0.33651 & & & \\
\hline
\end{tabular}

\begin{tabular}{|c|c|c|c|c|c|}
\hline \multicolumn{3}{|c|}{ Resumen del modelo } & & & \\
\hline$r^{2}$ & $\mathrm{r}^{2}$ ajustado & $\mathrm{r}^{2}$ pred. & & & \\
\hline $81.04 \%$ & $80.51 \%$ & $78.18 \%$ & & & \\
\hline \multicolumn{6}{|c|}{ Coeficientes } \\
\hline Término & Coef & EE del coef. & Valor T & Valor $\mathrm{p}$ & FIV \\
\hline Constante & -0.329 & 0.3 & -1.1 & 0.279 & \\
\hline Ln PBI & 1.0059 & 0.0811 & 12.4 & 0.000 & 1 \\
\hline
\end{tabular}

\section{Ecuación de regresión}

Ln C $=-0.329+1.0059$ Ln PBI

Estadístico de Durbin-Watson

$\operatorname{Ln} G=\beta_{0}+\beta_{1} \operatorname{Ln}$ PBI $_{P C}$

Donde Ln es el logaritmo natural.

La tabla 8 muestra los resultados de la estimación de la función logarítmica.

\section{DISCUSIÓN}

Coincidimos con Uribe (2000) quien considera que lo más importante es el que, en todos los países del mundo, la participación del estado ha estado en permanente crecimiento y, en esa situación, el problema, es como "volverlo eficiente y eficaz, cómo hacer que garantice el bienestar colectivo con el mínimo de costo, o diciéndolo de otra manera, cómo lograr una buena gobernabilidad, una gerencia pública de calidad total” (p. 85).
Pérez (2009) hace un análisis más bien cualitativo, pero su conclusión que "la ampliación del conjunto de bienes $\mathrm{o}$ servicios meritorios; es decir, el proceso político lleva a definir los niveles de consumo mínimo hasta llegar a un ámbito básico, al que da el carácter de necesidad" (p. 268), no contradice nuestros resultados. León (2017) encuentra también que en Colombia en el periodo 1970-2010, “el gasto público ha crecido significativamente frente al ingreso per cápita" (p. 871).

Campo y Mendoza (2018), se orientaron más bien a probar que, para el caso de Colombia, durante el periodo 1984-2012, opera la hipótesis Keynesiana llegando a la conclusión que hay un "efecto positivo y significativo del gasto público primario sobre el PIB” (p. 78).

Bulacio (2000) encuentra que en el período 1960-1999 el gasto público de Argentina tuvo una "la elasticidad parcial del gasto con respecto al ingreso estimada para el período de 0,87 " (p. 15), lo cual contradice la Ley de Wagner que requiere que la elasticidad sea mayor a 1 .

Coincidimos con Rodríguez, VenegasMartínez y Lima (2013), quienes en su estudio del caso mexicano durante el periodo 1950-2009" concluyen que el gasto público con el crecimiento económico "efectivamente cointegran. Es decir, 
guardan entre ellos una relación de largo plazo mediante trayectorias comunes" ( $p$. 92).

No coincidimos con Díaz y Revuelta (2009), quienes en su análisis de la ley de Wagner en España y América Latina desde 1960 hasta 2000, concluyen que, en España, "en lugar de la ley de Wagner, debemos aceptar la conjetura keynesiana, dado que es el incremento del gasto público el que causa el crecimiento económico" (p. 23) y, en las economías latinoamericanas, "la ley se cumple en catorce de los diecinueve estados, siendo las excepciones Perú, Chile, República Dominicana, El Salvador y Nicaragua" (p. 24).

Concordamos con Sánchez (2009) quien sostiene que la Ley de Wagner, se ha cumplido en el caso español, porque "la inversión pública en infraestructuras se situó en 2007 en el 3,8\% del Producto Interior Bruto, lo que representa el $147 \%$ de la media de la eurozona. Y 20 puntos de PIB más que la privada" (p. 1).

Nuestros resultados coinciden con lo encontrado por Jaén (2014) para el caso español durante el periodo 1960-1995, sobre lo cual concluye que considerando "el gasto público crecerá como consecuencia del crecimiento económico cualquiera que sea la forma en que éste se mida (aunque siempre en relación con el PIB)" (p. 29).
Discrepamos con Feijóo y Peralta (2017) quienes no han encontrado, en el caso de Perú para el periodo 1985-2015, evidencia del cumplimiento de la Ley de Wagner. Coincidimos con Aparco y Flores (2019) quienes verificaron el cumplimiento de la Ley de Wagner en el Perú, en el periodo comprendido entre 1950 y 2016, quienes validan en el largo plazo la Ley de Wagner en todas las versiones especificadas. Izquierdo, Puig, Riera-Crichton y Vuletin (2018) validan la ley de Wagner, en todo el mundo durante la década del 90, puesto que “a medida que aumenta el PIB per cápita, el gasto público tiende a elevarse, tanto en el margen extensivo (aparecen actividades y servicios nuevos) como en el margen intensivo (se amplían las actividades y servicios existentes)" (p. 1).

\section{CONCLUSIONES}

Finalmente, de acuerdo con la investigación realizada, podemos concluir lo siguiente:

De acuerdo con los resultados de las estimaciones $y$, a pesar de la implementación de una política económica ajustada a la propuesta contenida en el Consenso de Washington, el gasto público real ha continuado creciendo durante el periodo 1980-2018. 
De acuerdo con los resultados de las estimaciones, ha quedado demostrado que ha existido un impacto positivo del PBI real sobre el Gasto Público y que la elasticidad PBI del Gasto público real ha sido mayor que uno, durante el periodo 1980-2018.

De acuerdo con los resultados de las estimaciones, ha quedado demostrado que ha existido un impacto positivo del PBI real sobre el Consumo Público y que la elasticidad PBI del Consumo público real ha sido mayor que uno, durante el periodo 1980-2018.

De acuerdo con los resultados de las estimaciones, ha quedado demostrado que ha existido un impacto positivo del PBI per cápita real sobre el Gasto Público y que la elasticidad PBI per cápita del Consumo público real ha sido mayor que uno, durante el periodo 1980-2018.

En síntesis, entonces, las estimaciones prueban que, en las tres versiones analizadas, se cumple la Ley de Wagner en el Perú para el periodo 1980-2018.

\section{REFERENCIAS BIBLIOGRÁFICAS}

Aparco, E. y Flores, A. (2019). La hipótesis Keynesiana del gasto público frente a la Ley de Wagner: un análisis de cointegración y causalidad para Perú.
Revista de Economía del Rosario. Vol. 22. No. 1. Enero-Junio 2019. 5373

DOI:

http://dx.doi.org/10.12804/revistas.ur osario.edu.co/economia/a.7764

Boloña, C. (1993). Cambio de rumbo: el programa económico para los '90. Lima: Instituto de Economía de Libre Mercado - SIL.

Bulacio, J. M. (2000). La Ley de Wagner y el Gasto Público en Argentina. Universidad Nacional de Tucumán. Tucumán Argentina. Recuperado de https://aaep.org.ar/anales/pdf_00/BU LACIO.PDF

Campo, J. y Mendoza, H. (2018). Gasto público y crecimiento económico: un análisis regional para Colombia, 1984-2012. Lecturas de Economía, 88 (enero-junio), pp. 77-108 (C) Universidad de Antioquia, 2018. Recuperado de http://www.scielo.org.co/pdf/le/n88/ 0120-2596-le-88-00077.pdf

Díaz, D. y Revuelta, J. (2009). Gasto público y crecimiento en América Latina y España. XVI Encuentro de Economía Pública, Granada, 2009. Recuperado de https://dialnet.unirioja.es/descarga/art iculo/2942042.pdf 
Díaz, O. E. (2009). Gasto público en México: rehén de vaivenes políticos. Fuente : Fortuna, September 15, 2009. Recuperado de https://www.voltairenet.org/article16 2423.html

Feijóo, E. P. y Peralta, E. M. (2017). Relación entre el gasto público y el crecimiento económico. un estudio empírico para los países de la comunidad andina para el periodo 1985 - 2015. (Tesis de licenciatura. Universidad de Cuenca). Cuenca, Ecuador. Recuperada de https://dspace.ucuenca.edu.ec/bitstre am/123456789/27403/1/Trabajo\%20 de\%20Titulaci\%C3\%B3n.pdf

Gandhi, V. P. (1971). La Ley de Wagner sobre los gastos públicos: ¿la confirman los recientes estudios de análisis por secciones transversales? Revista de economía política. Madrid : Vol. 58.1971, p. 75-88 Recuperado de

https://www.econbiz.de/Record/laley-de-wagner-sobre-los-gastosp\%C3\%BAblicos-la-confirman-losrecientes-estudios-dean\%C3\%A1lisis-por-seccionestransversales-gandhi/10002428259

Gujarati, D. (2006). Principios de econometría. $\quad\left(3^{\mathrm{a}}\right.$ ed.). Madrid:
McGraw-Hill/Interamericana de España, S. A. U.

Izquierdo, A., Puig, P., Riera-Crichton, D. y Vuletin, G. (2018). El gasto público: de más a mejor. En: Izquierdo, A., Pessino, C. y Vuletin, G. (Editores). (2018). Mejor gasto para mejores vidas: cómo América Latina y el Caribe puede hacer más con menos. Banco Interamericano de Desarrollo. Recuperado de https://flagships.iadb.org/es/DIA201 8/capitulo-1-el-gasto-publico-demas-a-mejor

Jaén, M. (2014). La ley de Wagner: un análisis sintético. Universidad de Almería. Recuperado de https://www.researchgate.net/publica tion/28078680_La_ley_de_Wagner_ un_analisis_sintetico/link/0912f5057 3df64017f000000/download

León, A. (2017). Ley de Wagner en el caso colombiano, 1970-2010. II Congreso Virtual Internacional Desarrollo Económico, Social y Empresarial en Iberoamérica (Junio 2017). Recuperado de http://www.eumed.net/librosgratis/actas/2017/desarrolloempresarial/74-ley-de-wagner-en-elcaso-colombiano.pdf 
Molina, G. y Gantier, M. F. (2015). Ley de Wagner y Keynes: El caso de Bolivia. Instituto de Investigaciones SocioEconómicas (IISEC), Universidad Católica Boliviana https://www.econstor.eu/bitstream/10 419/176615/1/2015-10.pdf

Pérez, M. (2009). Necesidades, derechos y políticas públicas: una mirada desde la teoría económica. Revista de Economía Institucional, vol. 11, n. ${ }^{\circ}$ 20, primer semestre/2009, pp. 253269.

Perrotini, I. (2002). Pepe Ayala, la economía institucionalista y algunas palabras elegíacas. Economía Informa $\mathrm{N}^{\circ} 307$ mayo 2002. Recuperado de http://www.economia.unam.mx/publi caciones/reseconinforma/pdfs/307/11 \%20Perrotini\%20Institucionalismo.p df

Rodríguez, D. Venegas-Martínez, F. y Lima, V. (2013). La ley de Wagner versus la hipótesis keynesiana: el caso de México, 1950-2009. Investigación Económica, vol. LXXII, núm. 283, enero-marzo, 2013, pp. 69-98 Distrito Federal, México. Recuperado de http://www.scielo.org.mx/scielo.php? script=sci_arttext\&pid=S018516672013000100004
Sánchez, C. (2009). España y la Ley de Wagner. Por qué el modelo de Estado es un lastre para la recuperación. Recuperado de https://www.elconfidencial.com/arch ivo/2009/02/27/mientras_tanto_69_e spana_wagner_modelo_estado_lastre _recuperacion.html

Uribe, R. (2000). El gasto público: una reducción bien difícil. Semestre Económico vol. 3, No. 6 pp. 62-86. Recuperado de https://revistas.udem.edu.co/index.ph p/economico/article/view/1418

\section{Contacto:}

Dr. Alberto Valenzuela Muñoz albertovm50@hotmail.com 